\title{
A rate of spread index for fires in spinifex fuels
}

\author{
J.J. Sharples ${ }^{\mathrm{a}}$, A.M. Gill ${ }^{\mathrm{b}}$ and N.D. Burrows ${ }^{\mathrm{c}}$ \\ ${ }^{a}$ School of Physical, Environmental and Mathematical Sciences, UNSW Canberra. \\ ${ }^{\mathrm{b}}$ Fenner School of the Environment and Society, Australian National University. \\ ${ }^{c}$ Department of Parks and Wildlife, Western Australia. \\ Email: j.sharples@adfa.edu.au
}

\begin{abstract}
Fires in spinifex occur throughout arid and semi-arid parts of Australia and in some cases can affect large tracts of the landscape with associated environmental impacts. In response to this environmental challenge an empirical model for the prediction of fire spread rate in spinifex fuels has recently been developed, based on a number of experimental burns conducted in Western Australia.

In other research related to fires in grasslands, a simple rate of spread index for quasi-equilibrium fire spread was developed and, despite its simplicity, was shown to provide practically identical output to current operational grassland fire spread prediction models. This simple rate of spread index for grasslands conceptualises the rate of fire spread as wind speed divided by fuel moisture content, where fuel moisture content is estimated using a fuel moisture index $(F M I)$. Such a conceptualisation embodies the notion that fires will spread faster in windier conditions and in fuels that are drier. The rate of spread index, as it applied to grassfires, also incorporates a term that accounts for an intensity-dependent indraft that counters the prevailing winds at the fire line. As such, the rate of spread index can be viewed as a two parameter model for quasi-equilibrium fire spread.
\end{abstract}

In this paper we investigated the performance of the rate spread index when applied to the discrete spinifex fuels of arid and semi-arid Australia. The performance of the rate spread index was evaluated through the use of empirical data relating to fires in spinifex and through comparison with the existing spinifex fire spread model.

The results indicated that the rate of spread index, as it was applied to grassfires, was only able to account for $68 \%$ of the variation in the observed rates of spread. Multiplication of the rate of spread index by fuel cover improved it's predictive ability to $73 \%$, but this was still not as good as the existing spinifex model, which could account for $83 \%$ of the variation in the data. The main reason for this relatively poor performance of the rate of spread index was found to be due to the fact that the $F M I$ did a poor job of estimating the moisture contents of spinifex fuels. As such, we concluded that application of the FMI should be restricted to more temperate fuel types, for which it has been shown to work quite well.

An alternate form of the rate of spread index, using actual fuel moisture content rather than the FMI, was considered and found to produce much more accurate predictions. Indeed, when multiplied by fuel cover, this alternate rate of spread index was able to account for $85 \%$ of the variation in the observed rates of spread, thereby slightly outperforming the existing models for spinifex. The final version of the rate of spread index can be expressed as a function of fuel cover $c, 2 \mathrm{~m}$ wind speed $U$ and profile fuel moisture content $m$ :

$$
S(U, m, c)=37 c \frac{\max (1, U)}{m},
$$

with corresponding rates of spread well predicted by the model

$$
R^{*}(U, m, c)=1.5 S(U, m, c)+600 .
$$

These results have implications for the parsimony of fire behaviour models and demonstrate how conceptual and pedagogical simplifications can be incorporated into fire spread models with no practical loss in model performance. The results are also relevant to the possible unification of fire spread models across different fuel types.

Keywords: Fire spread, spinifex, wildfire, fire behaviour modelling 


\section{INTRODUCTION}

Spinifex grasslands dominate significant proportions of the arid and semi-arid parts of Australia; perennial hummock grasses cover some $43 \%$ of Western Australia (Burrows et al., 2009), and exist to varying degrees in the other mainland states and the Northern Territory. In contrast to other grasslands, such as those that dominate parts of southeastern Australia, spinifex grasses present a more discrete fuel array, with inidividual plants (hummocks) separated by bare ground (see Figure 1). In addition to their patchy coverage of the landscape, the physical structure of spinifex plants results in differences in the way fires propagate across spinifex dominated landscapes. In particular, the ability of a fire to propagate through a particular field of spinifex, or not, is determined by the distribution of spacings between individual plants, the hummock dimensions, their moisture content and meteorological conditions. Nevertheless, the extreme fire weather conditions that can occur over regions dominated by spinifex grasses can make them highly flammable. Figure 1 shows a spinifex fire burning in the Western Desert region.

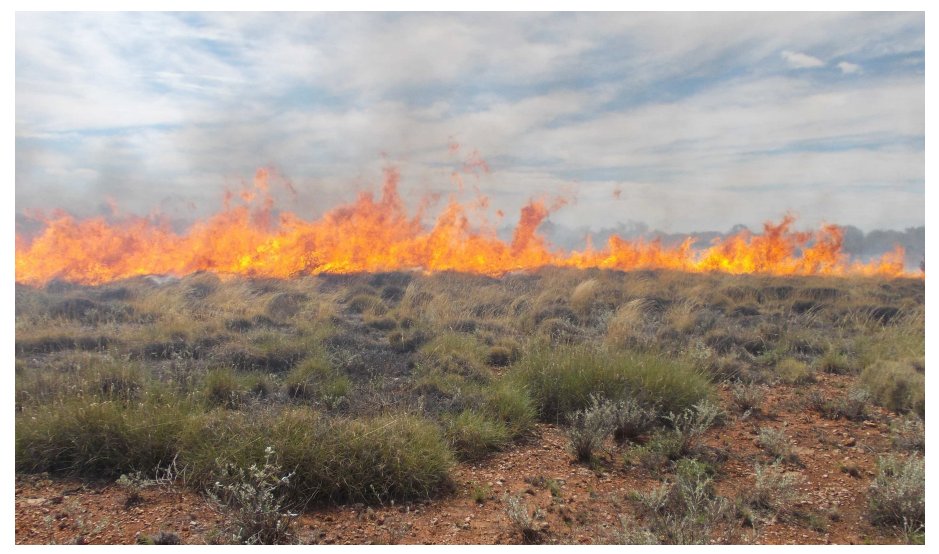

Figure 1. Fire propagating through spinifex grasslands in the Western Desert region. Photo: N.D. Burrows.

Burrows et al. (2009) cite evidence to support a change of fire regime in much of the spinifex grasslands of the Western Desert region, which now favours a simplified mosaic of infrequent, large wildfires. This change in fire regime, when combined with other influencing factors, constitutes a significant challenge to the management of biodiversity in such regions. Management strategies involving the use of prescribed fire to assist in the establishment of a finer-scale mosaic of burnt patches would appear to be a natural option. However, successful implementation of this option requires an understanding of fuel dynamics and fire behaviour within hummock grasses. As such, a number of authors have addressed the predictability of fire behaviour in spinifex fuels through development of empirical models relating to threshold conditions for fire propagation and for rate of spread.

Griffin and Allan (1984) developed a model to predict the rate of spread of fire in spinifex fuels based on experimental fires, which were conducted in the Northern Territory over a range of seasonal weather conditions and fuel ages. They determined that rate of spread could be described by an empirically-derived function of factors relating to weather (wind speed, air temperature and relative humidity) and the condition of the fuel (cover, patchiness and moisture content).

Using data obtained from an independent set of experimental fires, Burrows et al. (1991) found that the model of Griffin and Allan (1984) largely over predicted the rate of spread. Instead, they advocated a simpler model derived using linear regression analysis, which related rate of spread to wind speed, air temperature, fuel moisture content and fuel cover. This development was complemented by an additional set of experimental fires reported by Burrows et al. (2009), which were analysed in combination with those initially reported by Burrows et al. (1991). In addition to new regression models for rate of spread, Burrows et al. (2009) also considered thresholds for fire spread. These models will be described in more detail in the next section, as they will form the benchmark for the comparative analyses conducted in later sections of the paper.

Sharples and McRae (2013) introduced a simple index $S_{\alpha, \mu}$ for the rate of spread of grassland fires and compared its predictions with those arising from the CSIRO grassland fire spread model Cheney et al. (1998). The rate of spread index was found to give practically identical predictions of rate of spread, despite its far more parsimonious formulation. The rate of spread index of Sharples and McRae (2013) has two empirically 
derived parameters, $\alpha$ and $\mu$, in contrast to the CSIRO grassland fire spread model, which has sixteen empirically derived parameters for each of the two pasture types (natural and grazed) accommodated in the model. Moreover, the rate of spread index has a pedagogical advantage in that it can be simply conceptualised as 'wind speed divided by fuel moisture content'. As such, the rate of spread index embodies the intuitive notion that fires will spread more rapidly when winds are strong and when fuels are dry.

Given the success of $S_{\alpha, \mu}$ at emulating the predictions of the CSIRO grassland fire spread model, it is of interest to examine how the index performs when applied to other fuel types such as the spinifex grasslands discussed above. This is the main focus of this paper. The model of Burrows et al. (2009) will be used to provide benchmark predictions of rate of spread to which predictions derived from $S_{\alpha, \mu}$ will be compared. Predictions based on $S_{\alpha, \mu}$ and related indices will also be directly compared with observed rate of spread data.

\section{DATA AND METHODS}

\subsection{Fuel, fire weather and rate of spread data}

To facilitate comparison of the state-of-the-art rate of spread models of Burrows et al. (2009) with $S_{\alpha, \mu}$ and related indices we use data collected over the period 1988-2015 from a range of locations in the Western Desert and near Mt Isa (Williams et al., 2015). Most of these data are derived from experimental fires, including those reported by Burrows et al. (1991; 2009), though some data from operational burns are also included. The experimental fires were each ignited by a line of fire of lengths typically between $50 \mathrm{~m}$ and $100 \mathrm{~m}$. Most fires grew to a size of 1-2 ha, although some grew larger. Rates of fire spread $\left(\mathrm{m} \mathrm{hr}^{-1}\right)$ were determined by dividing the total forward distance propagated by the fire divided by the time taken to do so.

The weather data includes measurements of wind speed, air temperature and relative humidity, which have been averaged over the duration of the fire. Most fires (i.e. those that spread) burnt for around 5-10 minutes, with the odd fire burning for a longer period of time. Over the approximately 30 years of experiments, weather data has been collected from various heights ranging from 1.5-2.0 m above ground, or at eye level in the more recent cases. These small variations are not expected to significantly affect the ensuing analyses.

Spinifex is predominantly a live fuel with varying proportions of dead material, depending on its age. Fuel moisture content was determined by sampling a profile through the clump - taking live and dead material together. For each experimental fire, fuel cover was measured along a 100m continuous line transect by measuring the distance along the transect intercepted by live and dead fuel. The height of each intercepted live spinifex clump was also measured. Fuel load was determined by destructive sampling of five or ten $1 \mathrm{~m} \times 1 \mathrm{~m}$ plots, then converted to oven dry weight based on the measured values of profile moisture content.

In total the data set just described comprised 158 records. Of these data, 100 points represented cases where the fire successfully propagated, with the remaining 58 points corresponding to cases where the fire self extinguished. In this preliminary study we do not consider the problem of determining threshold burning conditions (colloquially referred to as go/no-go conditions), and so our attention is solely focused on the 100 cases of successful fire propagation.

\subsection{Spinifex fire spread model of Burrows et al. (2009)}

Burrows et al. (2009) reported empirical analyses of data arising from 83 experimental fires in spinifex fuels. Logisitic regression modelling was used to investigate the environmental conditions required for a fire to successfully propagate. These analyses resulted in the following propagation index, $P I_{F L}$ :

$$
P I_{F L}=0.57 U+0.96 w-0.42 m-7.42,
$$

where $U$ is the $2 \mathrm{~m}$ wind speed $\left(\mathrm{km} \mathrm{h}^{-1}\right), w$ is the fuel load $\left(\mathrm{t} \mathrm{ha}^{-1}\right)$ and $m$ is the fuel profile moisture (\%). The propagation index provides guidance on whether a fire will successfully propagate or self-extinguish. As discussed by Burrows et al. (2009), fires should be expected to spread when $P I_{F L}>0$, while for values of $P I_{F L} \leq-2$ fires are unlikely to spread. In cases where fires are expected to spread (i.e. when $P I_{F L}>0$ ), Burrows et al. (2009) determined that the rate of spread, $R_{F L}$, could be estimated by:

$$
R_{F L}=1581+154.9 U+140.6 w-228 m .
$$

They also determined an alternate model for the rate of spread in terms of fuel cover $c(\%)$ and average hummock height $h(\mathrm{~cm})$ :

$$
R_{F F}=1969+142.8 U-229.1 m+120.1 F F,
$$


J.J. Sharples, A.M. Gill and N.D. Burrows, A rate of spread index for fires in spinifex fuels

where $F F$ is the fuel factor defined as

$$
F F=0.25 c+0.04 h-0.32 .
$$

Burrows et al. (2009) state that $F F$ is almost equal to $w$ and so can be substituted for fuel load when direct field measurements are not available. For such situations, an alternate version of the propagation index is also given as:

$$
P I_{F F}=0.37 U-0.31 m+0.78 F F-5.23,
$$

with fires again only expected to spread when $P I_{F F}>0$ (i.e. $R_{F F}=0$ if $P I_{F F} \leq 0$ ).

\subsection{Indices for rate of fire spread in spinifex}

Sharples and McRae (2013) introduced a rate of spread index for grassland fires, which was defined using the FMI of Sharples et al. (2009) (after Pook and Gill (1993) who modelled moisture content of pine needles):

$$
F M I=10-0.25(T-H),
$$

where $T$ is temperature and $H$ is relative humidity. The $F M I$ has been shown to provide reasonable estimates of fuel moisture in some of the fuel types found in temperate regions of Australia (e.g. grasslands, eucalypt litter) (Sharples and McRae, 2013; Slijepcevic et al., 2013). It is of interest to note that $F M I$ is an approximate version of the fuel moisture submodel of the Mk3/4 grassland fire spread meters (McArthur, 1966).

In general, the rate of spread index takes the form of a two parameter family of functions that act on wind speed and FMI. The defining parameters $\mu$ and $\alpha$ are characterised as follows: $\mu$ represents an indraft factor, as it can be related to an indraft wind speed; while $\alpha$ can be considered as a calibration or scale factor. The rate of spread index itself is defined as follows:

$$
S_{\alpha, \mu}(U, F M I)=\alpha \frac{\max (1, U)}{F M I+\mu} .
$$

In addition to the rate of spread index defined in terms of $F M I$, we also consider a form defined in terms of the actual fuel moisture content $m$ :

$$
S_{\alpha, \mu}(U, m)=\alpha \frac{\max (1, U)}{m+\mu} .
$$

To better account for the important effects of fuel structure on rate of spread a number of different candidate models were also tested. Specifically, we considered simple multiplicative models such as $c S_{\alpha, \mu}, w S_{\alpha, \mu}$, $\operatorname{ch} S_{\alpha, \mu}$, etc. These were obtained by simply multiplying the indices (7) and (8) by the relevant fuel parameters.

\section{RESULTS}

\subsection{Performance of the models in predicting rate of spread}

Figure 2 shows the observed rates of spread plotted against the predictions of the $R_{F L}$ and $R_{F F}$ models of Burrows et al. (2009). Overall, these models perform quite well, with $R_{F L}$ and $R_{F F}$ both accounting for just over $83 \%$ of the variance in the rate of spread data. One shortcoming of the models is that in a number of instances they falsely predict zero rates of spread. This is particularly true of the $R_{F L}$ model. The $R_{F L}$ model predicts the observed rate of spread values with a mean absolute error of $451 \mathrm{~m} \mathrm{hr}^{-1}$ and a mean error (observed minus predicted) of $309 \mathrm{~m} \mathrm{hr}^{-1}$, which indicates a slight under prediction. The $R_{F F}$ model produced a mean absolute error of $387 \mathrm{~m} \mathrm{hr}^{-1}$ and a mean error of $-180 \mathrm{~m} \mathrm{hr}^{-1}$, indicating a slight over prediction of about $14 \%$ of the mean observed rate of spread. These results indicate that the $R_{F F}$ model is a slightly better predictor of rate of spread.

The performance of the rate of spread indices $S_{\alpha, \mu}(U, F M I)$ and $S_{\alpha, \mu}(U, m)$ are depicted in Figures 3(a) and 3(b). For the FMI based index (equation (7), figure 3(a)), the parameter $\alpha$ was estimated as $\alpha_{1}=1060$ by matching average values of the rate of spread index with the average of the observed values. The indraft factor was taken as $\mu=6$, as in Sharples and McRae (2013). For the $m$ based index (equation (8), figure 3(b)), the parameter $\alpha$ was estimated as $\alpha_{2}=1547$, again by matching mean values of index and observed values. For 
J.J. Sharples, A.M. Gill and N.D. Burrows, A rate of spread index for fires in spinifex fuels
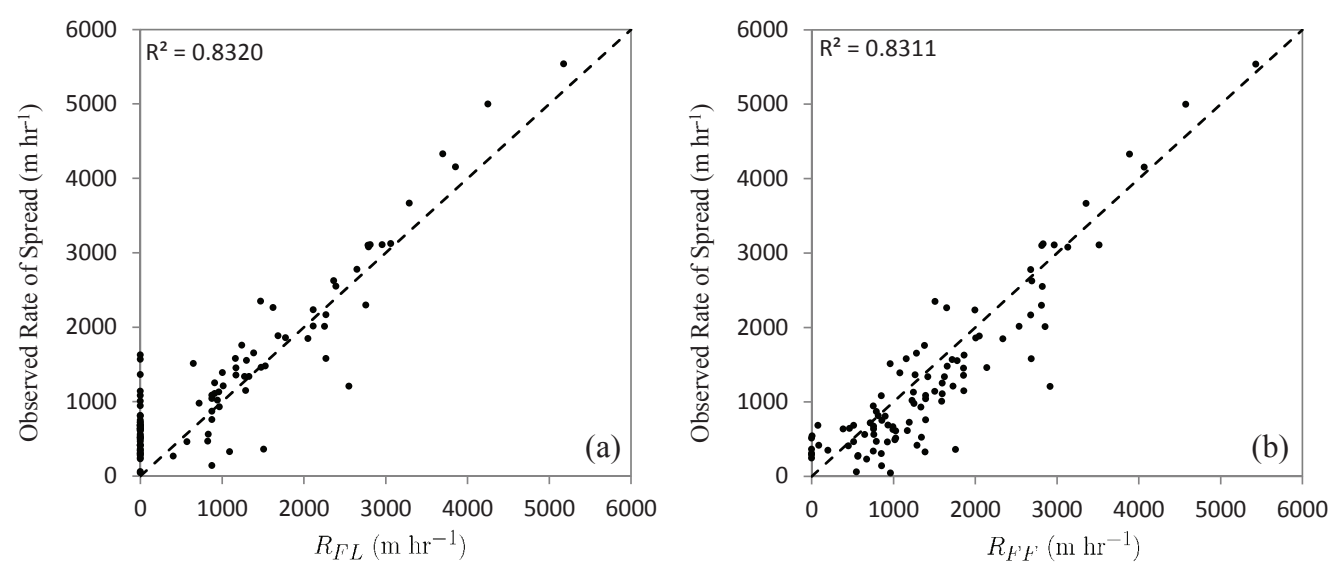

Figure 2. Observed rate of spread plotted against: (a) $R_{F F}$; and (b) $R_{F F}$. The corresponding $R^{2}$ values are given in each panel. The dashed lines indicate a perfect model fit.

this model the a more suitable value of the indraft factor was found to be $\mu=0$, though this parameter was not optimised quantitatively.

Overall the rate of spread indices performed reasonably well, though not as well as the models of Burrows et al. (2009). The FMI based index explained around $68 \%$ of the variance in the observed values, while the $m$ based index explained around $76 \%$ of the observed variance. Corresponding mean absolute errors were $457 \mathrm{~m}$ $\mathrm{hr}^{-1}$ for the FMI based index, and $420 \mathrm{~m} \mathrm{hr}^{-1}$ for the $m$ based index.

The models obtained by multiplying the rate of spread indices by the various fuel structure components did provide for better predictions in general, but the best improvement in predictive power was given by multiplying the indices by fuel cover $c$. The performance of these models are depicted in Figures 3(c) and 3(d). The model given by $c S_{\alpha_{3}, 6}(U, F M I)$, with $\alpha_{3}=26$, was found to explain around $73 \%$ of the variance in observed rate of spread, with a corresponding mean absolute error of $420 \mathrm{~m} \mathrm{hr}^{-1}$. The best predictive model, however, was found to be $c S_{\alpha_{4}, 0}(U, m)$, with $\alpha_{4}=37$. This model explained around $85 \%$ of the variance in observed rate of spread, with a corresponding mean absolute error of $393 \mathrm{~m} \mathrm{hr}^{-1}$, which is about the same as that for the $R_{F F}$ model. Figure 3 (d) indicates that while there is a very close linear relationship between the observed rates of spread and values of $c S_{\alpha_{4}, 0}(U, m)$, it is misaligned with the line of perfect fit. However, this is not really an issue; the results clearly indicate that for all practical intents, the model $c S_{\alpha_{4}, 0}(U, m)$ provides an equivalent scale for rate of spread. Indeed, the transformation defined by

$$
R^{*}(U, m, c)=1.5 c S_{\alpha_{4}, 0}(U, m)+600,
$$

produces a model that fits the observed rate of spread data with a mean absolute error of $325 \mathrm{~m} \mathrm{hr}^{-1}$ and a mean error of $-31 \mathrm{~m} \mathrm{hr}^{-1}$, which is approximately $-2 \%$ of the average observed rate of spread. The $R^{*}(U, m, c)$ model did produce a small number of negative rate of spread values, however.

\subsection{Intermodel comparisons}

Figure 4 shows how predictions of the two models of Burrows et al. (2009) compare with the $c S_{\alpha_{4}, 0}(U, m)$ index. The figure portrays a strong linear relationship between the predictions of both $R_{F L}$ and $R_{F F}$ with the rate of spread index $c S_{\alpha_{4}, 0}(U, m)$. This is particularly true for the $R_{F F}$ model, which exhibits an $R^{2}$ value of 0.89 with the rate of spread index. Again, the relationships between the models of Burrows et al. (2009) and $c S_{\alpha_{4}, 0}(U, m)$ do not follow the one-to-one lines, but this does not alter the fact that $c S_{\alpha_{4}, 0}(U, m)$ provides a measure of rate of spread that is practically equivalent to those provided by $R_{F L}$ and $R_{F F}$.

\section{DISCUSSION AND CONCLUSIONS}

A number of candidate indices for the rate of spread of fires burning in spinifex fuels were introduced and evaluated through their ability to predict observed rates of spread and through comparison with established spinifex rate of spread models (Burrows et al., 2009). Although the rate of spread index for grassland fires introduced by Sharples and McRae (2013) was found to accurately emulate the predictions of an established 
J.J. Sharples, A.M. Gill and N.D. Burrows, A rate of spread index for fires in spinifex fuels
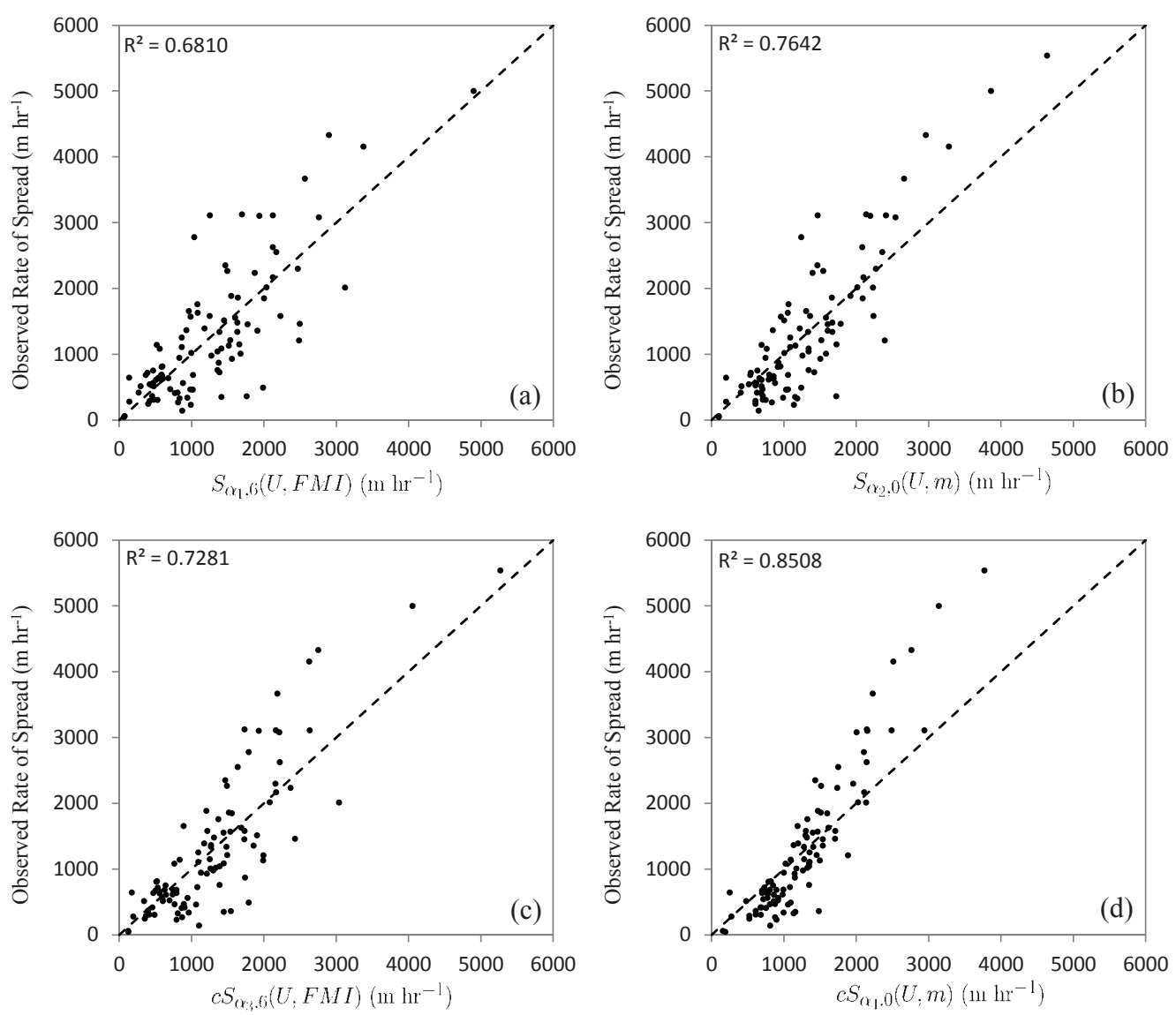

Figure 3. Observed rates of spread plotted against various rate of spread indices: (a) $S_{\alpha_{1}, 6}(U, F M I)$, $\alpha_{1}=1060$; (b) $S_{\alpha_{2}, 0}(U, m), \alpha_{2}=1547$; (c) $c S_{\alpha_{3}, 6}(U, F M I), \alpha_{3}=26$; (d) $S_{\alpha_{4}, 0}(U, m), \alpha_{4}=37$. The corresponding $R^{2}$ values are given in each panel. The dashed lines indicate a perfect model fit.

grassland fire spread model, it was not as successful in emulating the predictions of the models of Burrows et al. (2009) and was only able to account for about $68 \%$ of the variance in observed rates of spread. A likely reason for this relatively poor performance is the fact that the $F M I$ relates poorly to the observed profile moisture contents of spinifex fuels $\left(R^{2}=0.1\right)$. As such it appears that use of the FMI should be restricted to more temperate fuel types, for which it has been shown to perform well (see Slijepcevic et al. (2013) and Resco de Dios et al. (2015), for example).

Implementing the rate of spread index using measured fuel moisture content $m$ rather than $F M I$ resulted in better predictions of rate of spread, although this required alteration of the indraft factor to $\mu=0$. With these changes the rate of spread index was able to account for just over $76 \%$ of the variance in observed rates of spread. The best correspondences with observed rates of spread, however, were produced when the rate of spread indices were multiplied by fuel cover $c$. This is in contrast to the index used by Sharples and McRae (2013) to predict grassland fire rate of spread, which did not incorporate any dependence on fuel structure. This finding reflects the inherent differences in fire propagation mechanisms that exist in the continuous grasslands found in temperate regions and the patchy, discrete spinifex grasslands found in arid and semi-arid Australia.

Overall, the rate of spread index $c S_{\alpha_{4}, 0}(U, m)$ was found to produce the best correspondence with the observed rates of spread, accounting for around $85 \%$ of their variance and slightly outperforming the $R_{F F}$ model of Burrows et al. (2009). In addition to its slightly better predictive power, the rate of spread index offers a far more parsimonious and intuitive model for rate of spread. As such it offers a pedagogical advantage over existing models for predicting the rate of spread of spinifex fires. Moreover, the fact that an index with a very similar functional form can be successfully applied to both spinifex and temperate grasslands, suggests that a more universal approach to modelling the spread of fire across multiple fuel types may be a possibility. 
J.J. Sharples, A.M. Gill and N.D. Burrows, A rate of spread index for fires in spinifex fuels
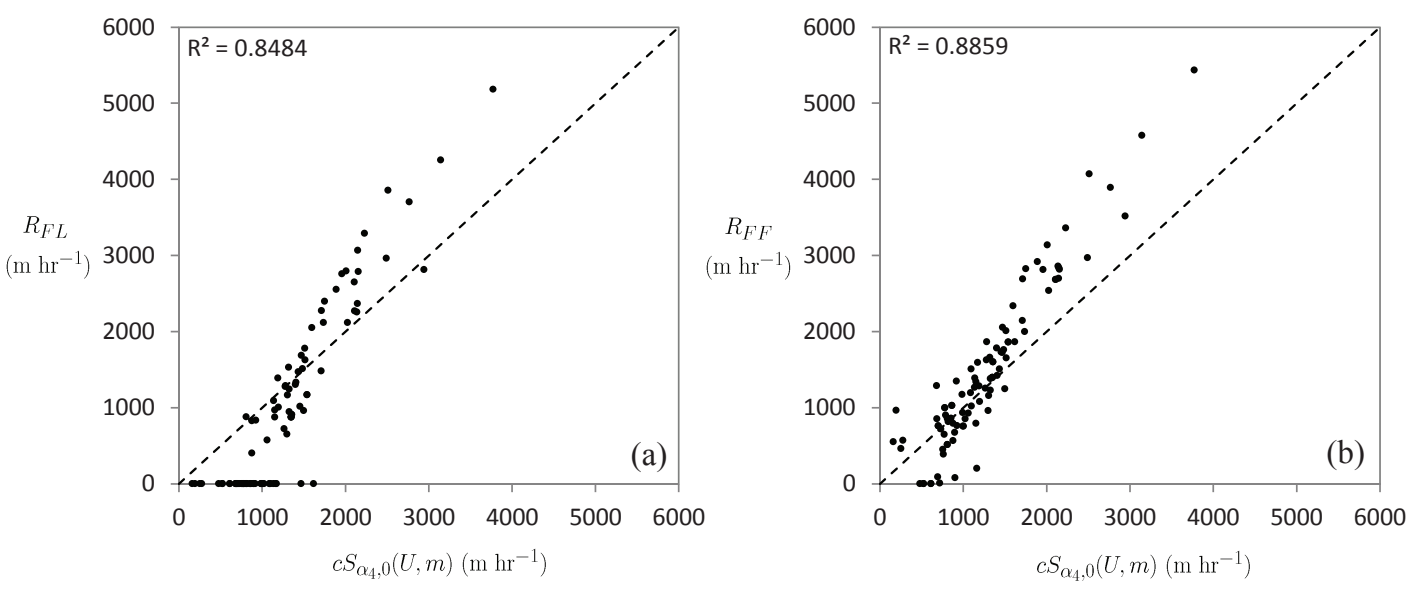

Figure 4. Predictions of the models of Burrows et al. (2009) plotted against $c S_{\alpha_{4}, 0}(U, m)$ : (a) $R_{F L}$; (b) $R_{F F}$. The corresponding $R^{2}$ values are given in each panel. The dashed lines indicate perfect agreement.

\section{ACKNOWLEDGEMENT}

The authors are indebted to Paul Williams from James Cook University for provision of his data and his permission to use it in this study. J.J. Sharples is supported by the Australian Research Council (IN130100038, IN140100011).

\section{REFERENCES}

Burrows, N., B. Ward, and A. Robinson (1991). Fire behaviour in spinifex fuels on the Gibson Desert Nature Reserve. Journal of Arid Environments 20, 189-204.

Burrows, N., B. Ward, and A. Robinson (2009). Fuel dynamics and fire spread in spinifex grasslands of the western desert. Proceedings of the Royal Society of Queensland 115, 69-76.

Cheney, N., J. Gould, and W. Catchpole (1998). Prediction of fire spread in grasslands. International Journal of Wildland Fire 8, 1-13.

Griffin, G. and G. Allan (1984). Fire behaviour. In E. Saxon (Ed.), Anticipating the inevitable: a patch burn strategy for fire management at Uluru (Ayers Rock-Mt Olga) National Park.

McArthur, A. (1966). Weather and grassland fire behaviour. Leaflet 100, Commonwealth Department of National Development, Forestry and Timber Bureau, Canberra, ACT.

Pook, E. and A. Gill (1993). Variation of live and dead fine fuel moisture in pinus radiata plantations of the australian capital territory. International Journal of Wildland Fire 3, 155-168.

Resco de Dios, V., A. Fellows, R. Nolan, M. Boer, R. Bradstock, F. Domingo, and M. Goulden (2015). A semi-mechanistic model for predicting the moisture content of fine litter. Agricultural and Forest Meteorology 203, 64-73.

Sharples, J. and R. McRae (2013). A fire spread index for grassland fuels. In J. Piantadosi, R. Anderssen, and J. Boland (Eds.), 20th International Congress on Modelling and Simulation, pp. 249-255. The Modelling and Simulation Society of Australia and New Zealand Inc.

Sharples, J., R. McRae, R. Weber, and A. Gill (2009). A simple index for assessing fuel moisture content. Environmental Modelling and Software 24, 637-646.

Slijepcevic, A., W. Anderson, and S. Matthews (2013). Testing existing models for predicting hourly variation in fine fuel moisture in eucalypt forests. Forest Ecology and Management 306, 202-215.

Williams, P., E. Collins, M. Blackman, C. Blackman, J. McLeod, L. Felderhof, L. Colless, K. Masters, S. Coates, and G. Sturgess, A. Martin (2015). The influence of ignition technique on fire behaviour in spinifex open woodland in semiarid northern australia. International Journal of Wildland Fire. In press. 